\title{
DRAFT DETC2007-35511
}

\section{INVESTIGATION OF BOUNDARY CONDITIONS FOR FLEXIBLE MULTIBODY SPACECRAFT DYNAMICS (DRAFT VERSION)}

\author{
John R. MacLean \\ An Huynh \\ METECS \\ Houston, Texas 77258 \\ Email: jmaclean@metecs.com, ahuynh@metecs.com
}

\author{
Leslie J. Quiocho* \\ NASA Johnson Space Center \\ Automation, Robotics, \& Simulation Division \\ Houston, Texas 77058 \\ Email: leslie.j.quiocho@nasa.gov
}

\begin{abstract}
In support of both the Space Shuttle and International Space Station programs, a set of generic multibody dynamics algorithms integrated within the Trick simulation environment have addressed the variety of on-orbit manipulator simulation requirements for engineering analysis, procedures development and crew familiarization/training at the NASA Johnson Space Center (JSC). Enhancements to these dynamics algorithms are now being driven by a new set of Constellation program requirements for flexible multibody spacecraft simulation. One particular issue that has been discussed within the NASA community is the assumption of cantilever-type flexible body boundary conditions. This assumption has been commonly utilized within manipulator multibody dynamics formulations as it simplifies the computation of relative motion for articulated flexible topologies. Moreover, its use for modeling of space-based manipulators such as the Shuttle Remote Manipulator System (SRMS) and Space Station Remote Manipulator System (SSRMS) has been extensively validated against flight data. For more general flexible spacecraft applications, however, the assumption of cantilevertype boundary conditions may not be sufficient. This paper describes the boundary condition assumptions that were used in the original formulation, demonstrates that this formulation can be augmented to accommodate systems in which the assumption of cantilever boundary conditions no longer applies, and verifies the approach through comparison with an independent
\end{abstract}

*Address all correspondence to this author. model previously validated against experimental hardware test data from a spacecraft flexible dynamics emulator.

\section{NOMENCLATURE}

$\Pi_{n} \quad$ Strain energy of body $n$

$q_{j}$ The $j^{\text {th }}$ generalized DOF of the system

$\dot{q}_{j} \quad$ First time derivative of $q_{j}$

$Q_{j} \quad$ The $j^{\text {th }}$ generalized force

$\vec{v}_{n}^{*} \quad$ Absolute velocity of point mass $d m$ inside body $n$

$\vec{a}_{n}^{*} \quad$ Absolute acceleration of $d m$ inside body $n$

$q_{n} \quad$ Flexible DOF of body $n$

$\dot{q}_{n} \quad$ First time derivative of $q_{n}$

$\ddot{q}_{n} \quad$ Second time derivative of $q_{n}$

$M_{r r, n} \quad$ Rigid-rigid mass matrix of body $n$

$M_{r e, n} \quad$ Rigid-elastic mass matrix of body $n$

$M_{e r, n} \quad$ Elastic-rigid mass matrix of body $n$

$M_{e e, n} \quad$ Elastic-elastic mass matrix of body $n$

$K_{e e, n} \quad$ Elastic stiffness matrix of body $n$

$F_{n p} \quad$ Force and moment of body $p$ acting on body $n$

$F_{n s} \quad$ Force and moment of body $s$ acting on body $n$

$r_{s} \quad$ Spatial vector from joint $n$ to joint $s$

$E_{r, n}$ External rigid forces and moments acting on body $n$

$E_{e, n} \quad$ External elastic forces and moments acting on body $n$

$B_{r, n} \quad$ Nonlinear rigid inertia force of body $n$

$B_{e, n} \quad$ Nonlinear elastic inertia force of body $n$

$S_{s} \quad$ Mode shape and mode slope of joint $s$ due to body $n$ flex 
$F_{n} \quad$ Force and moment from body $p$ (previous body to $n$ ) acting at joint $n$

$F_{s} \quad$ Force and moment from body $n$ (previous body to $s$ ) acting at joint $s$

\section{BACKGROUND}

In support of both the Space Shuttle and International Space Station programs, a set of generic multibody dynamics algorithms integrated within the Trick simulation environment have addressed the variety of on-orbit manipulator simulation requirements for engineering analysis, procedures development and crew familiarization/training at the NASA Johnson Space Center (JSC) [1,2]. Enhancements to these dynamics algorithms are now being driven by a new set of Constellation program [3] requirements for flexible multibody spacecraft simulation. One particular issue that has been discussed within the NASA community is the assumption of "cantilever-type" flexible body boundary conditions [4]. This assumption has been commonly utilized within manipulator multibody dynamics formulations as it simplifies the computation of relative motion for articulated flexible topologies. Moreover, its use for modeling of spacebased manipulators such as the Shuttle Remote Manipulator System (SRMS) and Space Station Remote Manipulator System (SSRMS) has been extensively validated against flight data. For more general flexible spacecraft applications, however, it has been pointed out that the assumption of cantilever-type boundary conditions may not be sufficient [5].

The purpose of this paper is to investigate the application of the Trick-based multibody dynamics algorithms for a specific case in which the assumption of cantilever boundary conditions no longer applies. It is shown that the formulation can be recast to accommodate more general boundary conditions without significant modifications or reduction in efficiency. To verify the approach, a case study is chosen that includes pinned end mass boundary conditions. The reformulated multibody dynamics model is then correlated against a transfer function model which has been previously validated against experimental hardware data obtained from a spacecraft flexible dynamics emulator.

\section{MULTIBODY DYNAMICS FORMULATION}

In [1], Modified Lagrange's Equations were developed to support the simulation of space-based manipulators such as the SRMS and SSRMS:

$$
\oint \frac{\partial\left(\vec{v}_{n}^{*}\right)^{T}}{\dot{q}_{j}} \vec{a}_{n}^{*} d m+\frac{\partial \Pi_{n}}{q_{j}}=Q_{j}
$$

Using Equation(1), it was shown that the EOMs in matrix form for body $n$, a single body from within a tree topology, are

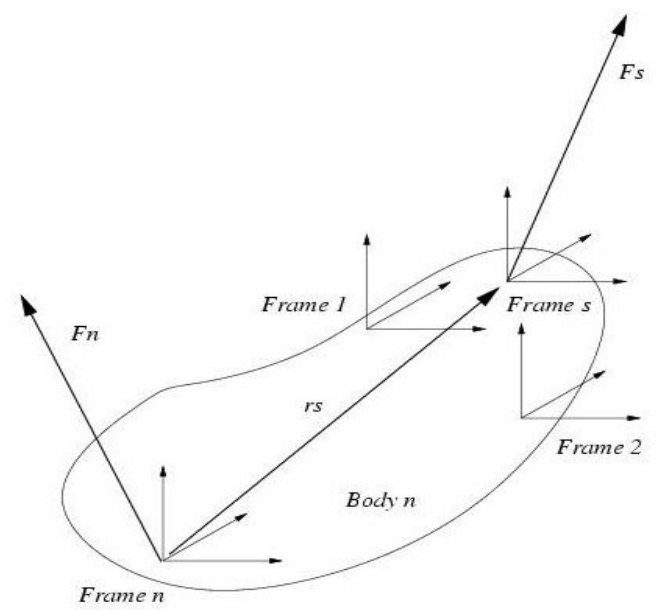

Figure 1. BODY N SCHEMATIC

$$
M_{r r, n} A_{n}+M_{r e, n} \ddot{q}_{n}=F_{n}-\sum_{s \neq n} r_{s} F_{s}+E_{r, n}+B_{r, n}
$$

$$
M_{e r, n} A_{n}+M_{e e, n} \ddot{q}_{n}+K_{e e, n} q_{n}=-\sum_{s \neq n} S_{s}^{T} F_{s}+E_{e, n}+B_{e, n}
$$

Equations 2 and 3 represent the core body level elements of the Trick-based multibody dynamics package, also known as MBDYN.

Figure 1 depicts a schematic representation of body $n$. Here the inboard frame is given as Frame $n$ while the outboard frame is shown as Frame s. $F_{n}$ represents the agregate force and moment vector of the previous body acting on body $n$ at Frame $n$; likewise $F_{s}$ is the force and moment vector of current body acting on the next body $s$ at Frame s. Referring back to Equation 3, it is important to notice that the $S_{n}^{T} F_{n}$ term corresponding to the mode shape and mode slope of Frame $n$ is not included. The absence of this particular term results from the cantilever-type boundary condition discussed in the previous section.

Extending these equations to the more general boundary condition (i.e., eliminating the cantilever-type assumption) results in

$$
M_{r r, n} A_{n}^{*}+M_{r e, n}^{*} \ddot{q}_{n}=F_{n}-\sum_{s \neq n} r_{s} F_{s}+E_{r, n}+B_{r, n}^{*}
$$




$$
M_{e r, n}^{*} A_{n}^{*}+M_{e e, n}^{*} \ddot{q}_{n}+K_{e e, n} q_{n}=-\sum_{s \neq n} S_{s}^{* T} F_{s}+E_{e, n}+B_{e, n}^{*}
$$

where

$$
\begin{gathered}
M_{e r, n}^{*}=M_{e r, n}-M_{r r, n} S_{n} \\
M_{e e, n}^{*}=M_{e e, n}-M_{e r, n} S_{n}-S_{n}{ }^{T} M_{r e, n}+S_{n}{ }^{T} M_{r r, n} S_{n} \\
S_{s}{ }^{* T}=S_{s}{ }^{T}-S_{n}{ }^{T} r_{s}
\end{gathered}
$$

It is important to note that the original form of the equations remains intact, although the definition of matrix expressions has now been 'enhanced' to eliminate the cantilever-type boundary condition limitation.

\section{CASE STUDY}

In order to test the proposed approach for handling more general boundary conditions, a satellite arm flexible dynamics emulator was modeled in MBDYN. Whereas the space robotics systems previously modeled using MBDYN are characterized by stiff links and high ratio gearboxes at the articulations, this emulator has a very flexible link driven by a brushless direct drive motor and hence is a good test case for implementation of a pinned boundary condition. In addition, the dynamics system is simple enough to be modeled analytically. This section describes the emulator, its relevant dynamics characteristics, and implementation of the model using MBDYN.

\section{Experimental Hardware}

As reported in [6], an experimental apparatus was designed for the Communications Research Center in Ottawa, Ontario Canada to serve as a satellite arm flexible dynamics emulator. This research was performed in support of the joint United States/Canada Mobile Satellite (MSAT) system program (as depicted in by the illustration in Figure 2). The emulator consisted of a hinged-free flexible stainless steel beam with a direct-drive DC torque motor at the hinged end and a tip mass and position sensor at the free end. The beam was free to rotate while the tip mass floated on an air bearing table made of granite. The tip

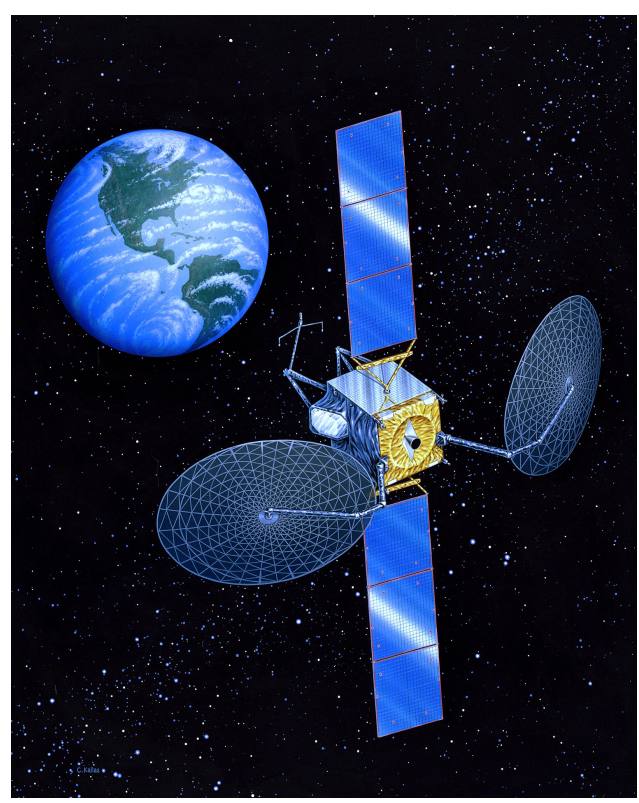

Figure 2. MSAT SATELLITE

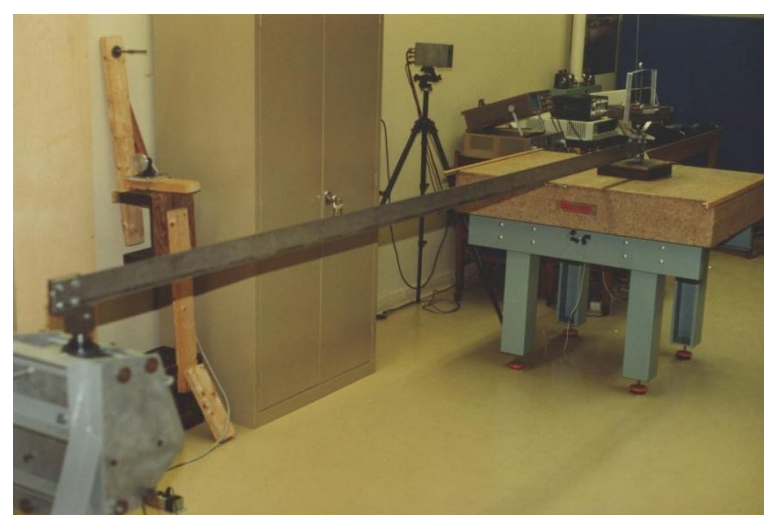

Figure 3. FLEXIBLE BEAM APPARATUS

mass was connected to the flexible beam via a pin type bearing. The output of the position sensor represented the transverse displacement of the tip from a reference position in the middle of the air bearing table. An additional sensor measuring the angular rotation of the motor shaft was also incorporated for experimentation. The apparatus is shown in Figure 3 while a schematic diagram representing the system as seen from above is provided in Figure 4.

Because of the high flexibility of the beam, the absence of a gearbox, and the analytically simple dynamics of the system, the emulator provides an excellent test case for MBDYN model implementation of a pinned boundary condition.

Unfortunately, as university lab space is frequently in short 


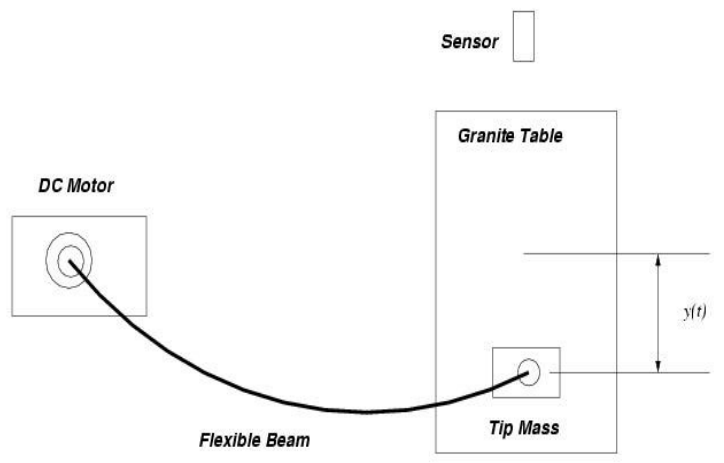

Figure 4. FLEXIBLE BEAM SCHEMATIC

supply, this apparatus has not existed for many years. An analytic model, however, was derived in [6] and was validated against the hardware test data, some of which still exists in the form of Matlab files. The analytic model, which is described below, makes no use of the cantilever-type assumption that restricted the MBDYN formulation and hence forms a suitable basis for testing the proposed MBDYN modeling approach.

\section{Analytic Emulator Model}

This section describes the analytic model that was developed in [6] and validated against the response of the emulator apparatus. As shown below the model makes no cantilever assumption.

Consider the classical Bernoulli-Euler beam equation [7]

$$
E I \frac{d^{4} y_{p}(x, t)}{d x^{4}}+\rho \frac{d^{2} y_{p}(x, t)}{d t^{2}}=0
$$

Using separation of variables, Equation (9) can be broken into two ordinary differential equations:

$$
\frac{E I}{\rho X(x)} \frac{d^{4} X(x)}{d x^{4}}-\sigma X(x)=0
$$

and

$$
\frac{d^{2} \Phi(t)}{d t^{2}}+\sigma \Phi(t)=0
$$

where $\sigma$ is a constant. The homogeneous solution for Equation(9) is given as
$X(x)=C_{1} \sinh \left(\frac{\mu}{l} x\right)+C_{2} \cosh \left(\frac{\mu}{l} x\right)+C_{3} \sin \left(\frac{\mu}{l} x\right)+C_{4} \cos \left(\frac{\mu}{l} x\right)$.

The four boundary conditions for the pinned end mass beam corresponding to the satellite arm emulator are

$$
X(0)=0
$$

$$
\frac{d^{2} X(l)}{d x^{2}}=0
$$

$$
l^{3} \frac{d^{3} X(l)}{d x^{3}}+\Xi \mu^{4} X(l)=0
$$

where $\mu^{4}=\frac{\rho \sigma l^{4}}{E I}$ and $\Xi$ represents the ratio between the tip mass and beam mass. For a stable solution we reject the case where $\sigma<0$ and we consider $\sigma=0$ and $\sigma>0$.

For the first case of $\sigma=0$,

$$
X_{o}(x)=A_{0} x
$$

and

$$
\Phi_{o}(t)=B_{0} t+C_{0}
$$

where $A_{0}$ is an arbitrary constant and $B_{0}, C_{0}$ are determined from the initial conditions.

For the second case of $\sigma>0$, it can be shown that there are an infinite number of solutions $\sigma_{i}, i=1,2,3 \ldots \infty$ such that

$$
\sigma_{i}=\frac{E I \mu_{i}^{4}}{\rho l^{4}}
$$




$$
\cot \mu_{i}=\operatorname{coth} \mu_{i}+2 \Xi \mu_{i}
$$

The solutions to Equations (10) and (11) are given as

$$
X_{i}(x)=A_{i}\left[\frac{\sinh \left(\frac{\mu_{i}}{l} x\right)}{\sinh \mu_{i}}+\frac{\sin \left(\frac{\mu_{i}}{l} x\right)}{\sin \mu_{i}}\right]
$$

and

$$
\Phi_{i}(t)=B_{i} \sin \left(\omega_{i} t+C_{i}\right)
$$

where

$$
\omega_{i}=\frac{\mu_{i}^{2}}{l^{2}} \sqrt{\frac{E I}{\rho}}
$$

Again, $A_{i}$ are arbitrary constants and $B_{i}, C_{i}$ depend on the initial conditions. The values $\omega_{i}$ represent the system modal frequencies while the functions $X_{i}(x)$, or 'shape' functions, are the system mode shapes.

It can be shown that these mode shapes, $X_{i}(x)$, are orthogonal with respect to the scalar product

$$
\left(X_{i}(x) \mid X_{j}(x)\right)=\int_{0}^{l} r(x) X_{i}(x) X_{j}(x) d x
$$

where

$$
\int_{0}^{l} r(x)=[1+\Xi l \delta(x-l)]
$$

and $\delta()$ is the Dirac delta function.

It is convenient to chose $A_{i}$ to normalize $X_{i}(x)$ (i.e., $\left.\left(X_{i}(x) \mid X_{j}(x)\right)=1\right)$. This results in the following values

$$
A_{0}=\sqrt{\frac{3}{l^{3}(1+3 \Xi)}}
$$

$$
A_{i}=\frac{1}{\sqrt{l\left(1+\Xi+2 \Xi \mu_{i}\left(\Xi \mu_{i}+\operatorname{coth} \mu_{i}\right)\right)}}
$$

Using virtual work techniques outlined in [6], the continuous time transfer functions from motor torque to tip position is given by

$$
P_{\text {tip }}(s)=\sum \frac{\frac{d X_{i}(0)}{d x}}{\rho} \frac{X_{i}(l)}{s^{2}+2 \zeta_{i} \omega_{i} s+\omega_{i}{ }^{2}}
$$

and the transfer function from motor torque to hub position is given by

$$
P_{h u b}(s)=\sum \frac{\frac{d^{2} X_{i}(0)}{d x^{2}}}{\rho\left(s^{2}+2 \zeta_{i} \omega_{i} s+\omega_{i}^{2}\right)}
$$

The contribution of the direct-drive torque motor with amplifier gain $K_{a}$, torque constant $K_{t}$, winding resistance $R$ and back EMF constant $K_{b}$ is given by

$$
P(s)=\frac{K_{a} K_{t} K_{s} P_{t i p}(s)}{R+K_{t} K_{b} s P_{h u b}(s)}
$$

Physical parameters of the system were determined by experimentation. They are listed in Table 1. Recall that $\Xi$ represents the ratio of the tip mass to mass of the flexible beam.

To validate the analytic model implementation, a sweep of sinusoidal forcing functions were applied to the system to identify the modal frequencies and these frequencies were compared to those derived theoretically from the model. This comparison, shown in Table 2, demonstrated a match of within $3.4 \%$.

The model was further verified by measuring the gain and the phase delay from input voltage to sensed tip position at a range of frequencies. The results shown in Figures 5 and 6 for frequencies from $0.1 \mathrm{~Hz}$ to $4 \mathrm{~Hz}$ demonstrate good agreement between analytic and hardware responses.

One can conclude, therefore, that the analytic emulator model, in the form of continuous time transfer functions, has been shown to be a good representation of the physical hardware. Based on the success of this comparison to hardware data, the parameters of the analytic model are used in the following section to derive input data for the more generalized MBDYN formulation which is then compared to the response of the analytic model. 


\begin{tabular}{clc}
\hline Parameter & Description & Value $($ SI units $)$ \\
\hline$E$ & Modulus of elasticity & N/A \\
$I$ & Area moment of inertia & N/A \\
$\rho$ & Linear density & $4.09 \times 10^{-4} \mathrm{lb}-\mathrm{sec} 2 / \mathrm{in} 2$ \\
& & $(2.82 \mathrm{~kg} / \mathrm{m})$ \\
$\sqrt{\frac{E I}{\rho}}$ & Combined beam properties & $9.3 \times 10^{3} \mathrm{in} 2 / \mathrm{sec}$ \\
& & $(5.974 \mathrm{~m} 2 / \mathrm{s})$ \\
$l$ & Length & $132.75 \mathrm{in}$ \\
& & $(3.372 \mathrm{~m})$ \\
$\Xi$ & Mass Ratio & 5.18
\end{tabular}

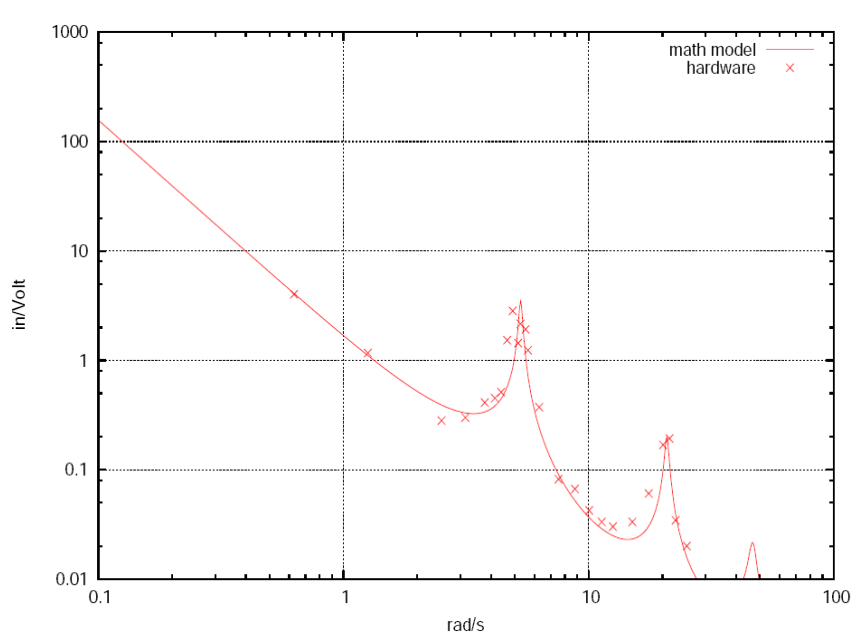

Figure 5. COMPARISON OF MAGNITUDE

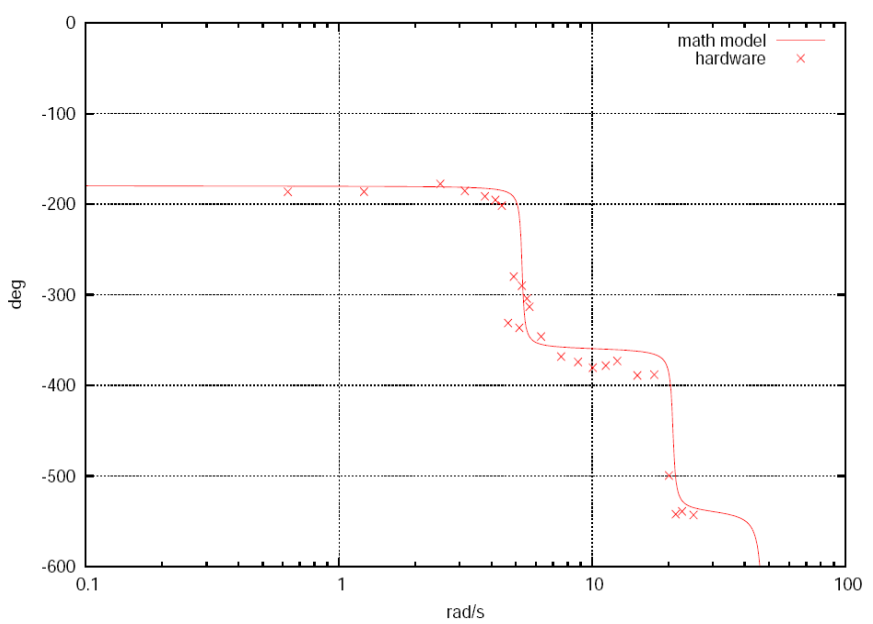

Figure 6. COMPARISON OF PHASE

Evaluating the terms of $M_{r e, n}$ for the problem at hand results in

$$
M_{r r, 1}=\left[\begin{array}{cc}
m_{1}[1] & -m_{1} \tilde{c}_{1} \\
m_{1} \tilde{c}_{1} & J_{1}
\end{array}\right]
$$

The rigid elastic coupling matrix is given as

$$
M_{r e, n}=\left[\begin{array}{c}
\int_{0}^{l} X_{i}(x) d m \\
\int_{0}^{l} \vec{r} \times X_{i}(x) d m
\end{array}\right]
$$

$$
\int_{0}^{l} X_{i}(x) d m=\frac{m A_{i}}{\mu_{i}}\left(\frac{\cosh \mu_{i}-1}{\sinh \mu_{i}}-\frac{\cos \mu_{i}-1}{\sin \mu_{i}}\right)
$$

and

$$
\int_{0}^{l} \vec{r} \times X_{i}(x) d m=\frac{A_{i}}{\sinh \mu_{i}}\left(\frac{l^{2}}{\mu_{i}} \cosh \mu_{i}-\frac{l^{2}}{\mu_{i}^{2}} \sinh \mu_{i}\right)
$$

Copyright (c) 2007 by ASME 


$$
+\frac{A_{i}}{\sin \mu_{i}}\left(-\frac{l^{2}}{\mu_{i}} \cos \mu_{i}+\frac{l^{2}}{\mu_{i}^{2}} \sin \mu_{i}\right)
$$

The elastic mass matrix can be represented by

$$
M_{e e, n}=\int_{0}^{l} X_{i}(x) X_{j}(x) d x
$$

Substituting $X_{i}$ from Equation ( 21) results in

$$
M_{e e, n}=-\left(\frac{m}{l}\right) 4 \Xi l A_{i} A_{j}
$$

for $i \neq j$ and

$$
M_{e e, n}=\left(\frac{m}{l}\right) \frac{1-3 \Xi+2 \Xi \mu_{i}\left(\Xi \mu_{i}+\operatorname{coth} \mu_{i}\right)}{1+\Xi+2 \Xi \mu_{i}\left(\Xi \mu_{i}+\operatorname{coth} \mu_{i}\right)}
$$

for $i=j$

The stiffness matrix can be found from

$$
K_{e e, n}=E I \int_{0}^{l} \frac{d^{2} X_{i}}{d x^{2}} \frac{d^{2} X_{j}}{d x^{2}} d x
$$

resulting in

$$
K_{e e, n}=E I\left(\frac{\mu_{i}}{l}\right)^{4}
$$

The lateral displacement as a function of distance along the beam, $x$ is given as

$$
y(x, t)=X(x) \phi(t)
$$

The mode slopes can then be calculated as

$$
\alpha_{i}=\frac{d X_{i}}{d x}=A_{i} \frac{\mu_{i}}{l}\left(\frac{\cosh \left(\frac{\mu_{i}}{l} x\right)}{\sinh \mu_{i}}+\frac{\cos \left(\frac{\mu_{i}}{l} x\right)}{\sin \mu_{i}}\right)
$$

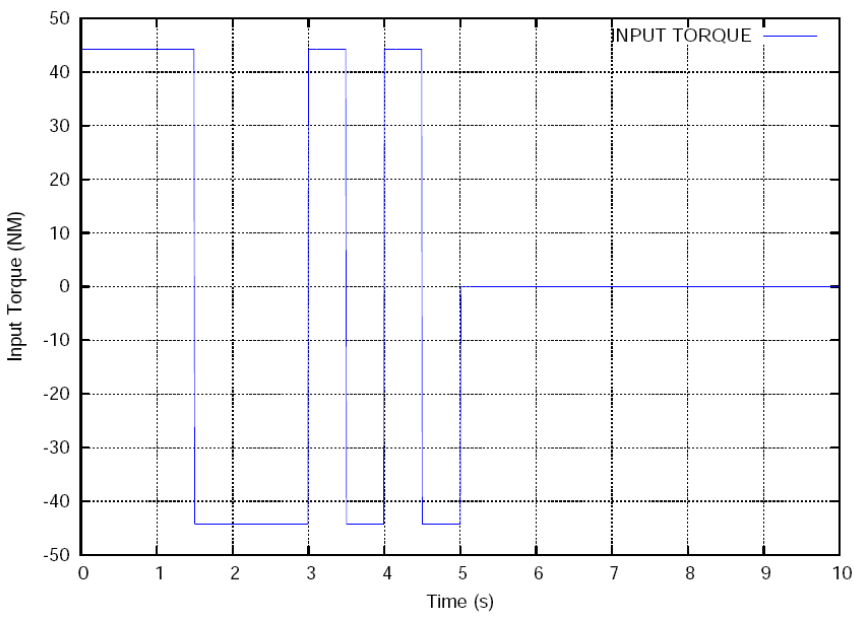

Figure 7. INPUT TORQUE

The resulting mode shape and slope matrix is therefore

$$
S_{s}(x)=\left[\begin{array}{l}
X(x) \\
\alpha(x)
\end{array}\right]
$$

The boundary condition at $x=0$ corresponds to a nonzero $S_{n}$ while the boundary condition at the tip corresponds to $S_{s}$. To implement the model in MBDYN, Equations (6), (7) and (8) are then used to calculate the required inputs.

\section{MODEL COMPARISON}

The next step of the process was to correlate the MBDYN implementation of the system model discussed previously against the original analytic dynamics model validated against the experimental hardware. To perform this model validation, a forcing function (see Figure 7) designed to excite the first two frequencies was employed and the resulting states for the hub and tip position were compared. Comparison plots are provided in Figures 8 and 10, while the difference plots are given in Figures 9 and 11. Note that an excellent comparison is achieved.

\section{CONCLUDING REMARKS}

To summarize, although originally formulated with the assumption of cantilever-type modes, it has been demonstrated through transformation of body level inputs that MBDYN is capable of handling more general boundary conditions using its existing mathematical formulation and implementation. Moreover, this capability has been verified through a case study by comparing to an analytical model (validated against test hardware) 


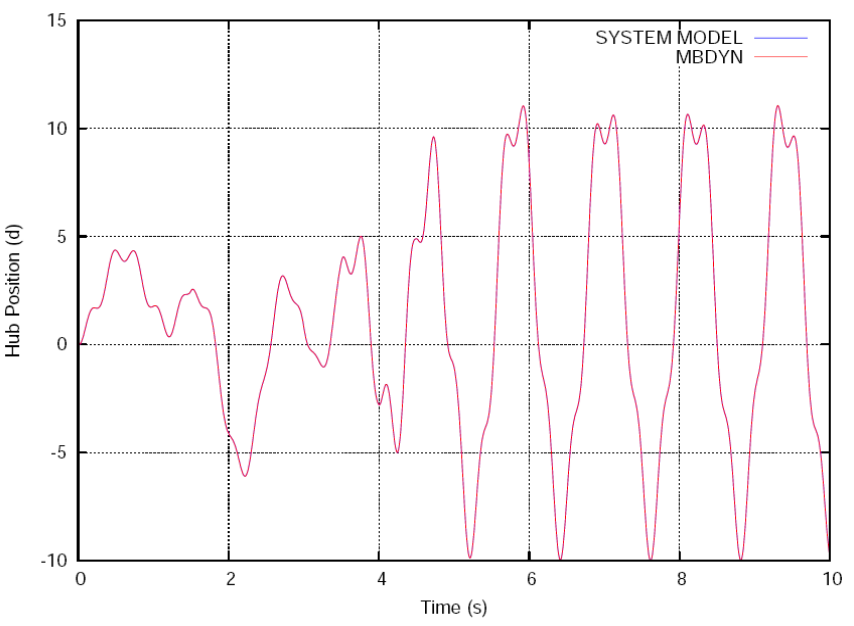

Figure 8. HUB POSITION COMPARISON

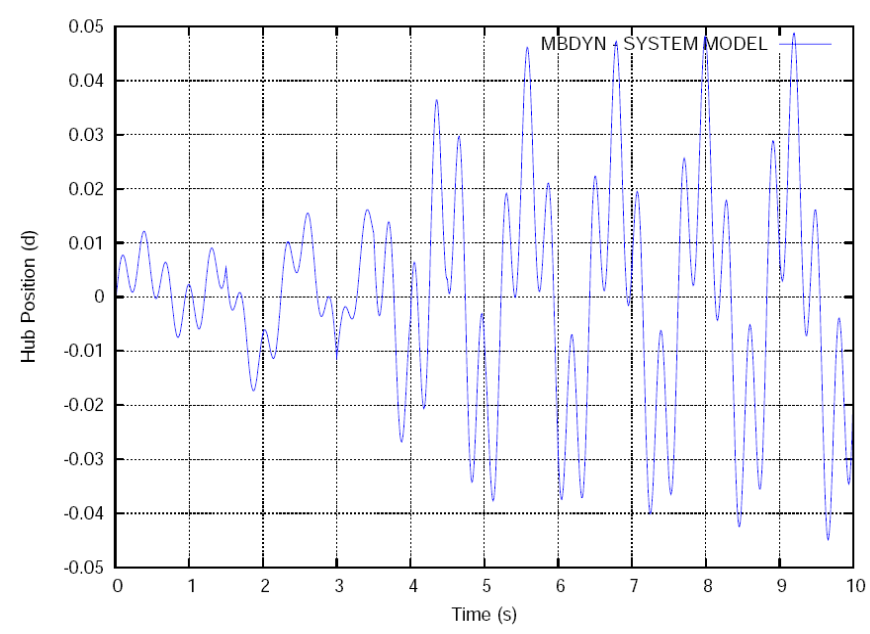

Figure 9. HUB POSITION DIFFERENCE

where the cantilever assumption no longer applies. Finally, it is anticipated that the approach described here within this paper can be extended to other scenarios involving flexible multibody spacecraft simulation with other types of boundary conditions.

\section{ACKNOWLEDGMENT}

The work described in this paper was performed entirely within the Simulation and Graphics Branch of the Automation, Robotics, and Simulation Division of the NASA JSC Engineering Directorate. The authors also wish to acknowledge Dr. Raymond Kwong of the University of Toronto for his technical oversight of the research associated with the modeling and testing of the satellite arm flexible dynamics emulator.

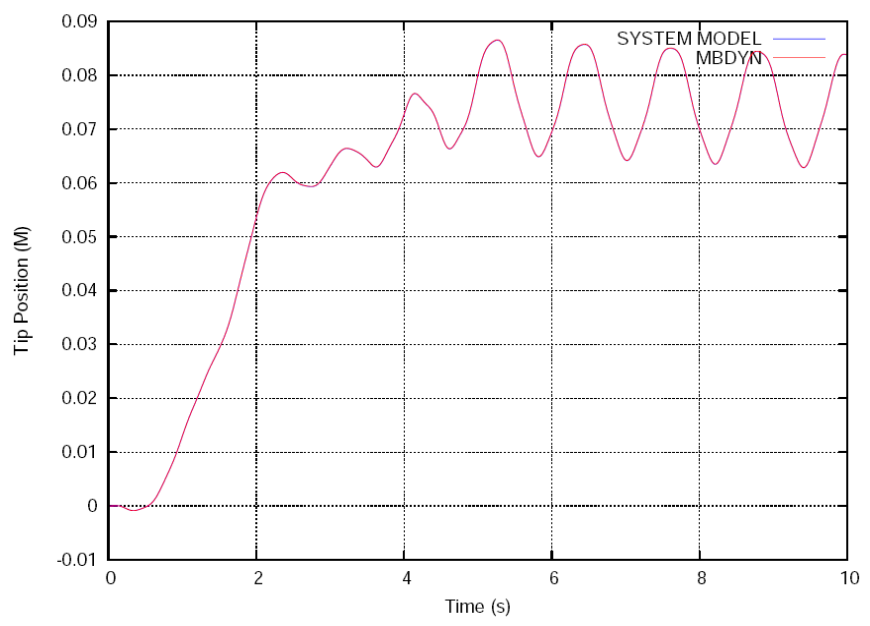

Figure 10. TIP POSITION COMPARISON

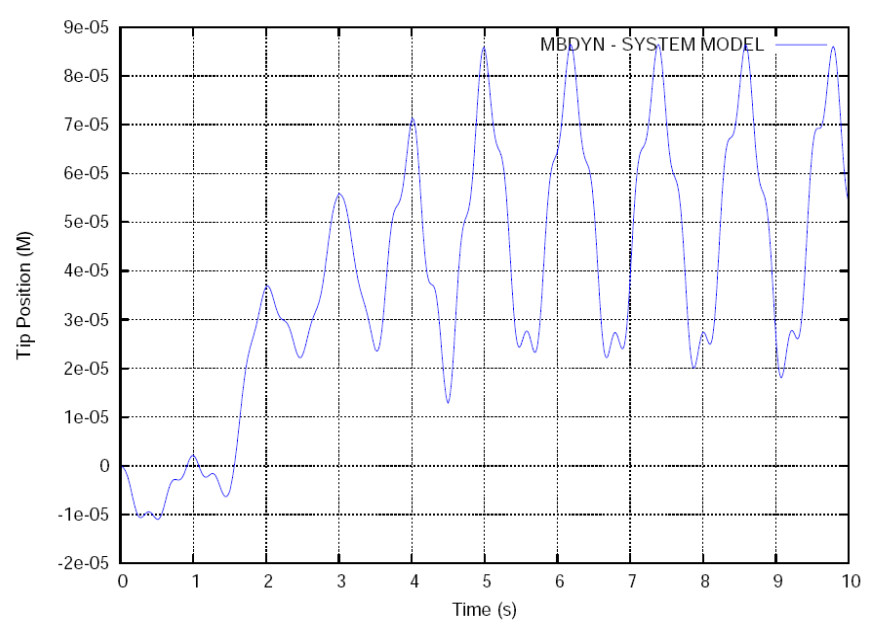

Figure 11. TIP POSITION DIFFERENCE

\section{REFERENCES}

[1] Quiocho, L.J., Huynh, A., Crues, E.Z, 2005, “Application of Multibody Dynamics to On-Orbit Manipulator Simulations", ASME 2005 International Design Engineering Technical Conferences \& Computers and Information in Engineering Conference, DETC2005-85545, Long Beach, CA.

[2] Paddock, E.J., Lin, A., Vetter, K., and Crues, E.Z., 2003, "Trick: A Simulation Development Toolkit", AIAA Modeling and Simulation Technologies Conference and Exhibit, AIAA 2003-5809, Austin, TX.

[3] NASA, 2005, NASA's Exploration Systems Architecture Study: Final Report, NASA-TM-2005-214062, Washington DC.

[4] Sincarsin, G.B., and Hughes, P.C., 1990, "Dynamics of 
Elastic Multibody Chains: Parts A - Body Motion Equations", Dynamics and Stability of Systems, Vol. 4., Nos. 3 \& 4, pp. 209-226.

[5] Tadikonda, S.S., Mordfin, T.G., and Hu, T.G., 1995, "Assumed Modes Method and Articulated Flexible Multibody Dynamics", Journal of Guidance, Control, and Dynamics, Vol. 18, No. 3, pp. 404-410.

[6] MacLean, J.R., 1990, Modeling and Control of a Flexible Beam, Master of Applied Science Thesis, Department of Electrical Engineering, University of Toronto, Toronto, Canada.

[7] Craig, R.R., 1981, Structural Dynamics: An Introduction to Computer Methods, John Wiley \& Sons, New York, NY. 